\title{
THE RANGE OF A VECTOR MEASURE HAS THE BANACH-SAKS PROPERTY ${ }^{1}$
}

\author{
R. ANANTHARAMAN
}

ABstract. The above result of Diestel and Seifert is proved using the Banach-Saks theorem for $L^{p}(\lambda), 1<p<\infty$.

Let $X$ be a real Hausdorff quasi-complete locally convex space (L.C.S.), $\mathbb{Q}$ be a $\alpha$-algebra of subsets of some set $S$ and $\nu: \mathbb{Q} \rightarrow X$ be a measure. We assume that $\nu$ is controlled, viz. that there exists a finite positive measure $\lambda$ on $\mathbb{Q}$ such that $\nu$ and $\lambda$ have the same null sets. When $X$ is a Banach space, Bartle, Dunford and Schwartz [3] proved every measure $\nu: \mathbb{Q} \rightarrow X$ to be controlled. This has been extended to a class of L.C.S. $X$, including metrizable ones, by Tweddle [8].

A set $A \subset X$ is said to have the Banach-Saks property if every sequence in $A$ has a subsequence $\left\{x_{n}\right\}$ such that the sequence of arithmetic means formed from $\left\{x_{n}\right\}$ converges. Using the methods of Szlenk [6], Diestel and Seifert [5] recently proved that $K=\overline{\operatorname{co}} \nu(\mathbb{Q})$ has the Banach-Saks property when $X$ is a Banach space. Our proof of an extension of this result is based instead on the Banach-Saks theorem for $L^{p}(\lambda), 1<p<\infty$ [2], [4, p. 78].

Since $X$ is quasi-complete, the set $K=\overline{c o} \nu(\mathbb{Q})$ is weakly compact by Theorem 3 of Tweddle [7]. Further, for each $\phi \in L^{\infty}(\lambda)$ the weak integral $\int \phi d \nu$ belongs to $X$ (see e.g. [1, Lemma 1]). Let $T: L^{\infty}(\lambda) \rightarrow X$ be the weak integral map, and let $T_{0}$ denote its restriction to the weak*-compact convex set

$$
P \equiv\left\{\phi \in L^{\infty}(\lambda): 0 \leqslant \phi(s) \leqslant 1 \text { s-a.e. }\right\} .
$$

Then $T_{0}(P)=K$.

As in Proposition 1 of [1] (proved there for $p=1$ ), the next result easily follows from Egoroff's theorem.

1. Proposition. Let $1<p<\infty$. Then the restriction of $T$ to every norm bounded subset $B$ of $L^{\infty}(\lambda)$ is continuous relative to the $L^{p}$-norm on $B$ and the given topology of $X$.

2. THEOREM. Let $\nu: \mathbb{Q} \rightarrow X$ be a controlled measure. Then $K=\overline{\operatorname{co}} \nu(\mathbb{Q})$ has the Banach-Saks property.

Received by the editors March 31, 1977.

AMS (MOS) subject classifications (1970). Primary 28A45.

'This research was supported by a grant from NRC, Canada. 
Proof. Let $\left\{x_{n}\right\}$ be a sequence in $K$. Since $K$ is weakly compact, by Eberlein's theorem we may replace $\left\{x_{n}\right\}$ by a subsequence $\left\{x_{m}\right\}$ that converges weakly to some $x \in K$. Since $T(P)=K$, for every $m$ there exists $\phi_{m} \in P$ such that $T\left(\phi_{m}\right)=x_{m}$. Fix $p, 1<p<\infty$. As $P$ is a bounded subset of $L^{p}(\lambda)$, by the Banach-Saks theorem there exists a subsequence $\left\{\phi_{i}\right\}$ of $\left\{\phi_{m}\right\}$ such that the sequence $\left\{\psi_{i}\right\}$ formed of arithmetic means of $\left\{\phi_{i}\right\}$ converges to some $\psi \in L^{p}(\lambda)$ in the norm of $L^{p}$. Clearly, $\psi \in P$. By Proposition 1 and the linearity of $T$ the sequence $\left\{T\left(\phi_{m}\right)\right\}$, viz. $\left\{x_{m}\right\}$ has arithmetic means that converge to $T(\psi) \in T(P)=K$. Since $\left\{x_{m}\right\}$ converges weakly to $x$, we have $T(\psi)=x$, which completes the proof.

\section{REFERENCES}

[1] R. Anantharaman, On exposed points of the range of a vector measure, Vector and Operator Valued Measures and Applications (Proc. Sympos. Snowbird Resort, Alta, Utah, 1972), Academic Press, New York, 1973, pp. 7-22.

[2] S. Banach and S. Saks, Sur la convergence forte dans les champs $L^{p}$, Studia Math. 2 (1930), 51-57.

[3] R. G. Bartle, N. Dunford and J. T. Schwartz, Weak compactness and vector measures, Canad. J. Math. 7 (1955), 289-305.

[4] J. Diestel, Geometry of Banach spaces-Selected topics, Springer-Verlag, New York, 1975.

[5] J. Diestel and C. J. Seifert, An averaging property of the range of a vector measure, Bull. Amer. Math. Soc. 82 (1976), 907-909.

[6] W. Szlenk, Sur ies suites faiblement convergentes dans l'espace L, Studia Math. 25 (1966), 337-341.

[7] I. Tweddle, Weak compactness in locally convex spaces, Glasgow Math. J. 9 (1968), 123-127. [8] , Vector-valued measures, Proc. London Math. Soc. 20 (1970), 469-489.

Department of Mathematical Sciences, Lakehead University, Thunder Bay, Ontario, CANADA 